\title{
DIRECT 3D STYLIZATION PIPELINES
}

\section{Contact:}

artineering.io/projects/Maya-Watercolor

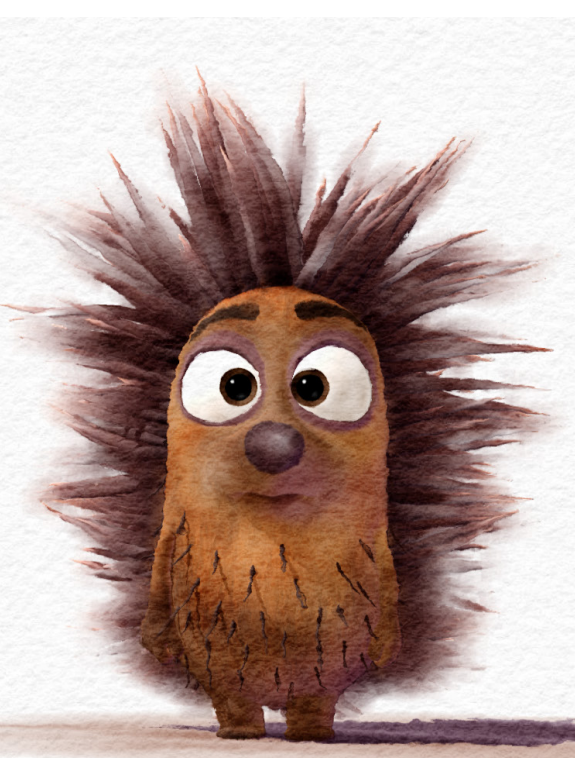

Using 3D computer graphics to emulate watercolor presents a special challenge. Complex stylizations are commonly processed offline, by combining multiple passes in compositing, where art directing is slow and non-intuitive because the stylized result is not immediate.

This direct 3D stylization pipeline allows art direction to happen in real time. Using the framework, artists can assign their desired local and global effects directly in the 3D scene, see the stylized results immediately, and intuitively adapt them to fit their stylized vision.

The technique can be used and applied in 3D animations, games, VR, visualizations, illustrations, and interactive art.

This research is supported by the National Research Foundation, Prime Minister's Office, Singapore, under its IDM Futures Funding Initiative.

Santiago Montesdeoca

Hock Soon Seah

Davide Benvenuti

Nanyang Technological University

Hans-Martin Rall

Nanyang Technological University
Pierre Bénard

INRIA, Université de Bordeaux, CNRS

Joëlle Thollot

Romain Vergne

Université Grenoble Alpes, INRIA, CNRS 